\title{
Silver halide sensitized gelatin derived from BB-640 holographic emulsion
}

\author{
Cristian Neipp, Inmaculada Pascual, and Augusto Beléndez
}

\begin{abstract}
Silver halide sensitized gelatin (SHSG) is one of the most interesting techniques for the production of holographic optical elements, achieving relatively high sensitivity of photographic material with a low scattering of dichromated gelatin. Here we present experimental results for SHSG derived from the novel BB-640, a red-sensitive ultra-fine-grain emulsion from Holographic Recording Technologies (Steinau, Germany). The material is characterized before recording and after processing, and information about the thickness, absorption, and refractive-index modulation of the final holograms is obtained. The influence of the developer is analyzed, and diffraction efficiencies as great as $96.2 \%$ (after allowing for reflections) with a transmission of $1 \%$ and absorption and scatter losses of $2.8 \%$ are obtained with AAC developer. Our investigations reveal that high-quality SHSG transmission holograms may be obtained with the new BB-640 plates. (C) 1999 Optical Society of America
\end{abstract}

OCIS codes: $\quad 090.2900,090.7330,230.1950,040.5190$.

\section{Introduction}

Silver halide sensitized gelatin (SHSG) has proved to be a good alternative to dichromated gelatin (DCG) for the production of transmission holograms. SHSG is a hybrid process that combines the sensitivity of photographic emulsion with the quality of DCG. In this processing the exposed emulsion is developed, bleached, and fixed, and the silver halide grains are eliminated from the emulsion. The recorded holographic image is due to the variation in the degree of hardening between the exposed and the nonexposed zones of the emulsion.1,2 During the bleaching process, the developed silver is oxidized to $\mathrm{Ag}^{+}$, whereas the $\mathrm{Cr}^{+6}$ ions are reduced to $\mathrm{Cr}^{+3}$ during the same bleaching bath. These $\mathrm{Cr}^{+3}$ ions form cross links between the gelatin chains in the vicinity of the oxidized silver grains, causing local hardening. After the fixing step, the processing is completed with the washing and the dehydration of the emulsion by

C. Neipp and A. Beléndez (augusto@disc.ua.es) are with the Departamento de Física, Ingeniería de Sistemas y Teoría de la Señal, Universidad de Alicante, Apartado 99, E-03080 Alicante, Spain. I. Pascual is with the Departamento Interuniversitario de Optica, Universidad de Alicante, Apartado 99, E-03080 Alicante, Spain.

Received 17 August 1998; revised manuscript received 24 November 1998.

0003-6935/99/081348-09\$15.00/0

(C) 1999 Optical Society of America means of successive baths in isopropanol, which is similar to the processing of DCG.

This technique was initially developed by Pennington and Harper, ${ }^{3}$ although the technique in its present state is the result of a study done by Graver et al. ${ }^{4}$ on the one hand and by Chang and Winick ${ }^{5}$ on the other. Ferrante ${ }^{6}$ studied the modulation transfer function closely and achieved a significant decrease in diffraction efficiency at 2000 lines $\mathrm{mm}^{-1}$, because the resolution possible with this technique is limited by the grain size of the photographic emulsion. $^{2}$ Hariharan ${ }^{7}$ analyzed the influence of the developing and bleaching bath and investigated the formation mechanism of the latent image in SHSG processing. He found that the above-mentioned processing scheme for SHSG is not the only mechanism involved and that the oxidation products of the developer, which have a local tanning action, also contribute to the formation of the latent image. Angell ${ }^{8}$ optimized the processing and the control of shrinkage for the Kodak $649 \mathrm{~F}$ emulsion, and significant improvements in diffraction efficiency over previous methods were found. Weiss and Millul ${ }^{9}$ worked out a simpler alternative SHSG processing method based on Hariharan's experiments and with the introduction of the CW-C2 developer into the SHSG process. Weiss and Friesem ${ }^{10}$ presented a relatively simple theoretical model for understanding the mechanism of SHSG hologram formation. In this model the storage of holographic gratings is a result of three separate contributions that are due to the silver ha- 
lide grains, the gelatin matrix, and voids in the gelatin matrix. With the exception of the work of Ferrante, Kodak 649F emulsions were used in these studies on SHSG holograms. In recent years some optimized procedures for SHSG holograms derived from Agfa plates have been published, ${ }^{11,12}$ showing that this emulsion is suitable for obtaining SHSG transmission holograms. An important contribution was made in a study by Phillips et al..$^{13}$ in which two new processing procedures for SHSG holograms were formulated. Although the Agfa emulsion is more difficult to use for SHSG holograms because it is thinner and has a gelatin type different than that of the Kodak $649 \mathrm{~F}$ emulsion, transmission gratings with diffraction efficiencies as great as $80 \%{ }^{11,12}$ and reflection gratings with diffraction efficiencies as great as 55\%14 can be produced with Agfa emulsions. High-quality holographic optical elements such as holographic collimators, holocouplers, and fan-infan-out devices have been made with this technique by use of Agfa emulsions. ${ }^{15,16}$

One of the photographic emulsions most frequently used in holography was produced by Agfa Gevaert. Agfa emulsions have been highly regarded over the past few decades. The fact that Agfa holographic materials will no longer be produced is of great concern to many in holography. ${ }^{17}$ Apparently, it is necessary to find and to study alternative silver halide materials, in particular, for SHSG holograms. One of the available silver halide materials is the new and unfamiliar BB-640 emulsion from Holographic Recording Technologies (Steinau, Germany) produced by Birenheide. ${ }^{18,19}$

This paper presents experimental results for SHSG transmission holograms recorded in BB-640 holographic plates. This study presents a modified formula for processing SHSG derived from BB-640 plates, and measurements of the diffraction efficiency and the transmission of these holograms and their absorption and scatter are analyzed and discussed. Using Kogelnik's theory, we obtained information about some holographic characteristics of diffraction gratings recorded as SHSG holograms in BB-640 plates, and the results are compared with those obtained by use of Agfa 8E75 HD plates processed under the same experimental conditions as with BB-640 plates. These results show that BB-640 emulsion is a good candidate for replacing the Agfa 8E75 HD emulsion for SHSG holograms and has higher diffraction efficiency, higher spatial-frequency response, and lower absorption and scatter than the Agfa material.

\section{Characteristics of BB-640 Emulsion}

\section{A. Plates Used in Experiments}

The material used in our experiments was BB-640, an ultra-fine-grain photographic emulsion suitable for holography. The material is in the form of a thin layer of emulsion on a 2.5-in. $\times 2.5$-in. glass backing $(1 \mathrm{in} .-2.54 \mathrm{~cm})$. The parameters of the glass were established as $n_{\text {glass }}=1.53 \pm 0.01$ for the refractive index (measured with reflectivity data), $\alpha_{\text {glass }}=$ $0.015 \pm 0.001 \mathrm{~mm}^{-1}$ for the absorption coefficient (measured with the transmission data of the glass substrate), and $d=1.83 \pm 0.01 \mathrm{~mm}$ for the thickness.

\section{B. Silver Halide Grain Size}

The photographic emulsion consists of microscopic grains of silver halide, predominantly silver bromide, suspended in a gelatin matrix. One of the most important characteristics of BB-640 emulsion is that the silver halide grains are smaller than those of Agfa $8 \mathrm{E} 75 \mathrm{HD}$ and Kodak $649 \mathrm{~F}$ plates. The size of the silver-halide grains of BB-640 emulsion is, following the manufacturer, between 20 and $25 \mathrm{~nm},{ }^{18}$ whereas the sizes of the grains in the case of Agfa 8E75 HD and Kodak $649 \mathrm{~F}$ plates are $44^{20}$ and $57 \mathrm{~nm},{ }^{21}$ respectively. Owing to the smaller size of the silver halide grains of BB-640 emulsion, scattering in holograms formed with BB-640 emulsion will be less than in holograms recorded in Agfa and Kodak plates. Conversely, the energetic sensitivity of BB-640 plates is less than that of Agfa 8E75 HD and Kodak 649F emulsions, since with smaller grains it is necessary to increase exposure time compared with larger grain emulsions.

In addition, the scattering produced during the recording of holograms will also be less for BB-640 emulsion than for Agfa 8E75 HD and Kodak 649F emulsions. As a result of the granular structure of photographic emulsion, scattering of the incident light occurs when holograms are recorded. This scattering effect limits both the resolution (related to the capacity to store interference fringes with high spatial frequencies) and the signal-to-noise ratio (related to the quality of the hologram obtained). ${ }^{22}$ Phillips et al..$^{20}$ analyzed the scattering in photographic emulsions, introducing the concept of scatter cross section $\sigma$ to denote Rayleigh scatter and considering that the grains' collectivity contributes to the concept of a mean-free-path scatter $L$; thus

$$
L=(1 / N \sigma)
$$

where $N$ is the number of silver halide grains per unit volume of the emulsion layer. To take into account the effect of this collectivity on the scattering of a large number of silver halide grains distributed over the gelatin base, Phillips et al. introduced the parameter $f_{s}$ as a quotient of the mean-free-path of scatter $L$ and the thickness $d$ of the emulsion layer. This parameter $f_{s}$ may be used as a figure of merit to characterize the emulsion layer. When $f_{s}$ is greater than the thickness $d$, it is possible to store holograms of high quality with high spatial frequencies, whereas the resolution of the material is poor if $f_{s}$ is the same or less than $d$. The parameter $f_{s}$ is given by ${ }^{20}$

$$
f_{s}=(1 / N \sigma d)
$$


Table 1. Numerical Values of the Parameters $\sigma, L$, and $f_{s}$ for Agfa $8 E 75$ $\mathrm{HD}$ and BB-640 Emulsions $(\lambda=633 \mathrm{~nm})$

\begin{tabular}{lccr}
\hline \multicolumn{1}{c}{ Emulsion } & $\sigma\left(\mu \mathrm{m}^{2}\right)$ & $L(\mu \mathrm{m})$ & $f_{s}$ \\
\hline Agfa 8E75 HD & $78 \times 10^{-7}$ & 26 & 4 \\
BB-640 & $1.2 \times 10^{-7}$ & 906 & 85 \\
\hline
\end{tabular}

The scattering effect of the emulsion is less at high values of $f_{s}$. For the scatter cross section Phillips also obtained the equation ${ }^{20}$

$$
\sigma=\frac{\pi}{12}\left(\frac{2 \pi}{\lambda}\right)^{4} \frac{n_{g}{ }^{4}\left(n_{h}{ }^{2}-n_{g}{ }^{2}\right)}{\left(n_{h}{ }^{2}+2 n_{g}{ }^{2}\right)^{2}} \delta^{6},
$$

where $\lambda$ is the wavelength in air, $n_{g}$ is the refractive index of gelatin $\left(n_{g}=1.54,{ }^{23}\right), n_{h}$ is the refractive index of the silver halide grains (for silver bromide, $n_{\mathrm{AgBr}}=2.236,{ }^{23}$ ), and $\delta$ is the diameter of the grains. From basic math it follows for the mass of silver bromide per unit area of the emulsion $m_{\mathrm{AgBr}}$ that

$$
m_{\mathrm{AgBr}}=\frac{d N \pi \delta^{3} \rho}{6}
$$

where $\rho$ is the density of silver bromide $(\rho=6.47 \mathrm{~g}$ $\mathrm{cm}^{-3}$ ). From Eq. (4) it follows for $N$ that

$$
N=\frac{6 m_{\mathrm{AgBr}}}{d \pi \delta^{3} \rho} .
$$

For Agfa 8E75 HD emulsion, $\delta \approx 44 \mathrm{~nm}, m_{\mathrm{AgBr}}=$ $8.7 \mathrm{~g} \mathrm{~m}^{-2}, 23$ and $d=6 \mu \mathrm{m},{ }^{24}$ whereas for BB-640 emulsion, $\delta \approx 22 \mathrm{~nm}, m_{\mathrm{AgBr}}=3.5 \mathrm{~g} \mathrm{~m}^{-2}, 18$ and $d=$ $10.7 \mu \mathrm{m}$ (this value was obtained by application of the coupled-wave theory as described in Section 4). From Eq. (5) we obtain $N=5.0 \times 10^{15}$ grains cm $\mathrm{cm}^{-3}$ for Agfa 8E75 HD emulsion and $N=9.2 \times 10^{15}$ grains $\mathrm{cm}^{-3}$ for BB-640 emulsion. Table 1 shows the values of the scatter cross section $\sigma$, the meanfree path for scatter $L$, and the figure of merit $f_{s}$, for the wavelength of the He-Ne laser $(\lambda=633 \mathrm{~nm})$ for both Agfa 8E75 HD and BB-640 emulsions. As can be seen in Table 1 , the numerical value of $f_{s}$ for BB640 emulsion is 20 times greater than for Agfa 8E75 $\mathrm{HD}$ emulsion. Moreover, for BB-640 emulsion $L$ is 85 times larger than the thickness $d$. This indicates on the one hand that the scattering during recording will be much less with BB-640 emulsion than with Agfa 8E75 HD emulsion and on the other that the resolution of the first emulsion will be higher than that of the second. These two facts are a clear indication of the high quality of BB-640 plates.

\section{Degree of Hardness of the Gelatin of BB- 640 Emulsion}

Another important characteristic is that the gelatin of the BB-640 emulsion is much harder than that of the Agfa emulsion. ${ }^{18,19}$ Consequently, distortion of the interference planes during processing is avoided. However, for the recording of holograms in SHSG it is necessary to soften the gelatin to obtain higher dif-

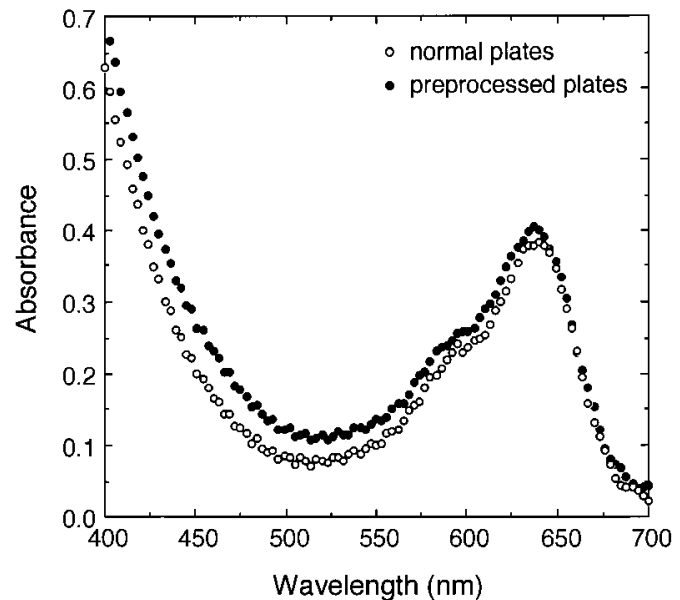

Fig. 1. Absorbance curves for BB-640 emulsion with and without pretreatment.

fraction efficiencies. ${ }^{1}$ Previous experiments carried out with BB-640 plates gave poor values of diffraction efficiencies for SHSG holograms recorded in BB-640 plates that were not pretreated before exposure to soften the gelatin of the emulsion. In our experiment the plates were hypersensitized before exposure to soften the gelatin of the emulsion, and the temperature of the bleaching bath was raised to $70{ }^{\circ} \mathrm{C} .25$

\section{Initial Experimental Considerations}

\section{A. Hypersensitization of the Plates}

As we indicated above, BB-640 plates have a low sensitivity, owing to the small grain size. This sensitivity may be increased by means of hypersensitization consisting of treating the photographic plate before exposure. The method we used was to immerse the plates in a solution of sodium sulphite and urea in distilled water. ${ }^{14}$ Sodium sulphite has a slight solvent effect on silver halides, whereas urea softens the gelatin, which as we explained is quite hard in the case of BB-640 plates and consequently is less suitable for SHSG processing. Below is a list of steps in the hypersensitization process:

1. Soak plates in $1 \%$ (in weight) sodium sulfite and $5 \%$ (in weight) urea solution at $20^{\circ} \mathrm{C}$ for $10 \mathrm{~min}$.

2. Rinse in running water for $1 \mathrm{~min}$.

3. Dry at $20^{\circ} \mathrm{C}$ and relative humidity $(\mathrm{RH})=60 \%$ for $24 \mathrm{~h}$.

We verified that the diffraction efficiency of holograms in SHSG with hypersensitized plates is greater than in nonhypersensitized plates. ${ }^{25}$

\section{B. Spectral Sensitivity of BB-640 Plates}

The spectral range of BB-640 plates is between 580 and $650 \mathrm{~nm}$. Figure 1 shows absorbance curves for both normal and hypersensitized plates with the procedure described above. Absorbance curves were 

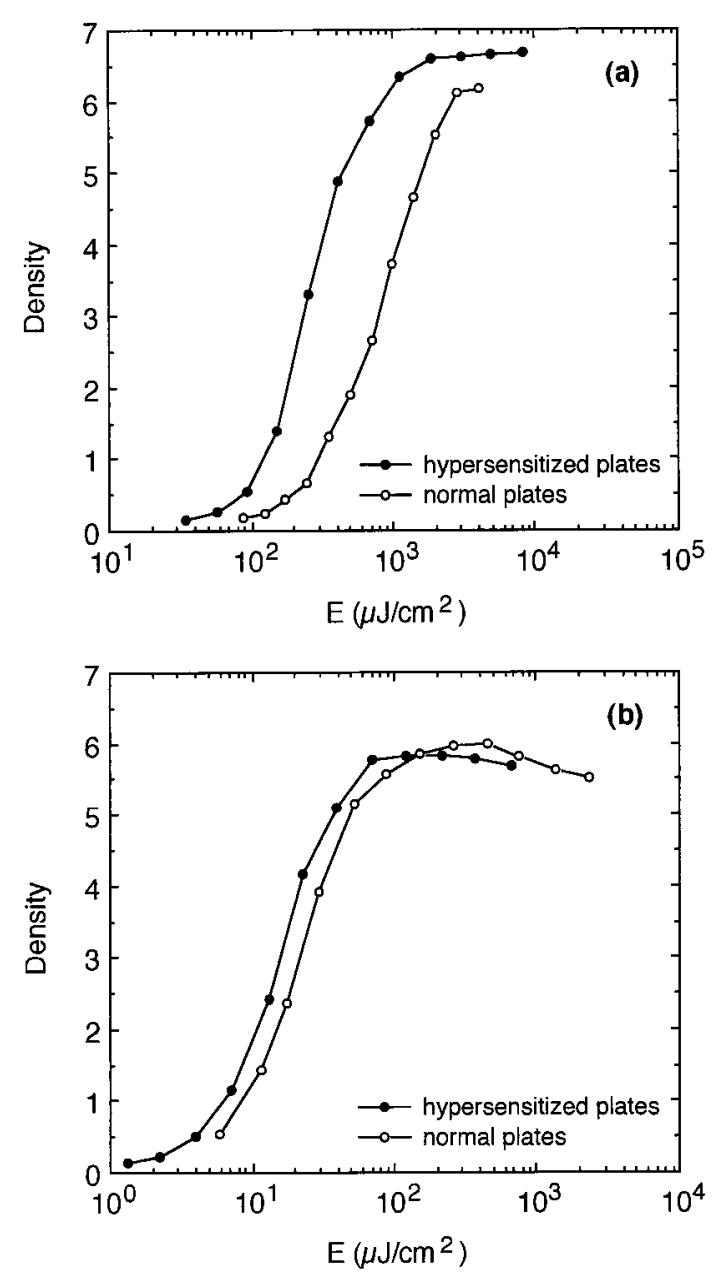

Fig. 2. $D-\log E$ curves for (a) BB-640 and (b) Agfa 8 E75 HD emulsions with and without pretreatment. D-19 developer.

obtained with the aid of a spectrophotometer. It can be seen that pretreatment produces a slight increase in the absorbance.

\section{Energetic Sensitivity of BB-640 Plates}

Figure 2 shows the $D$-log $E$ curves for BB-640 and Agfa 8E75 HD emulsions for both for hypersensitized and normal plates. We obtained the curves by recording absorption holograms by means of the interference of two collimated beams, symmetrically mounted, that formed an angle of $45^{\circ}$ with each other. We used the light from an $\mathrm{He}-\mathrm{Ne}$ laser polarized perpendicular to the plane of incidence. With this geometry, transmission gratings of $\sim 1200$ lines $\mathrm{mm}^{-1}$ were obtained. The incident intensity was $500 \mu \mathrm{W} \mathrm{cm}{ }^{-2}$, and the time of exposure was varied, twelve holograms being recorded in each case. After exposure all the plates were developed for $5 \mathrm{~min}$ at $20^{\circ} \mathrm{C}$ with D-19 developer. They were then washed in running water for $1 \mathrm{~min}$ and fixed with nonhardening F-24 fixer for $3 \mathrm{~min}$ at $20^{\circ} \mathrm{C}$. Next, the plates were washed in running water for $10 \mathrm{~min}$ and dried in a dessicator at low humidity $(<18 \%$ relative hu- midity) for $24 \mathrm{~h}$. Density $D$ was obtained in terms of transmittance $T$ as

$$
D=\log _{10}(1 / T),
$$

where transmittance $T$ was calculated as the ratio of light transmitted through the material and light incident on the material. The values of $T$ were corrected for reflection losses. Measurement of transmitted and incident light were carried out with the aid of a He-Ne laser illuminating the plates with a collimated beam entering normal to the surface of the plate. A magnitude that gives information about the energetic sensitivity of a photographic emulsion is the parameter $S$, defined as the value of the exposure $E$ corresponding to an amplitude transmittance of 0.5 , which means that the density $D$ is 0.6. ${ }^{26}$ As can be seen in Fig. 2(a), hypersensitization produces an increase in the energetic sensitivity and a decrease in the value of the parameter $S$. For BB-640 plates, $S$ decreases by a factor of 2.5 from 214

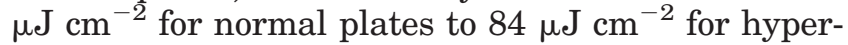
sensitized plates. Moreover, in the case of hypersensitized plates there is an increase in the contrast (slope of the linear zone of the $D-\log E$ curve). For hypersensitized Agfa 8E75 HD plates the increase in the energetic sensitivity is lower than for BB-640 plates, as can be seen in Fig. 2(b), since $S$ decreases only from $5 . \mu \mathrm{J} \mathrm{cm}^{-2}$ for normal plates to $4.5 \mu \mathrm{J} \mathrm{cm}^{-2}$ for hypersensitized plates. Also, a slightly higher maximum density is achieved with BB-640 plates than with Agfa 8E75 HD plates.

\section{Comparison of BB-640 with Agfa 8E75 HD Plates}

Unslanted phase transmission gratings were recorded on each plate by use of two collimated beams from a 15-mW He-Ne laser (633 nm), thus forming an angle of $45^{\circ}$ (in air) with each other. With the geometry described, the spatial frequency of the gratings was calculated as 1200 lines $\mathrm{mm}^{-1}$. The total intensity was $\sim 500 \mu \mathrm{W} \mathrm{cm}{ }^{-2}$, and the beam intensity ratio was 1:1. The exposed plates were processed according to the processing schedule shown below, and details of the developer and bleach formulas are given in Table 2 . The processing schedule is as follows:

A. Hypersensitization

B. Exposure

C. Development (All solutions are at $20^{\circ} \mathrm{C}$ except the bleaching step.)

1. Developer (5 min for D-19 developer; $4 \mathrm{~min}$ for AAC developer).

2. Rinse in running water for $2 \mathrm{~min}$.

3. Bleach for $30 \mathrm{~s}$ after the plate has cleared at $70{ }^{\circ} \mathrm{C}$.

4. Rinse in running water for $2 \mathrm{~min}$.

5. Soak in the nonhardening fixer Kodak F-24 for $2 \mathrm{~min}$.

6. Wash in running water for $10 \mathrm{~min}$.

7. Dehydrate in $90 \%$ isopropanol for $3 \mathrm{~min}$.

8. Dehydrate in $90 \%$ isopropanol for $3 \mathrm{~min}$.

9. Dehydrate in $100 \%$ isopropanol for $3 \mathrm{~min}$. 
Table 2. Developer and Bleach Formulas

\begin{tabular}{lc}
\hline \multicolumn{1}{c}{ Formula } & Amount \\
\hline D-19 Developer & \\
Metol & $2 \mathrm{~g}$ \\
Sodium sulfite (anhydrous) & $45 \mathrm{~g}$ \\
Hydroquinone & $8 \mathrm{~g}$ \\
Sodium carbonate (anhydrous) & $50 \mathrm{~g}$ \\
Potassium bromide & $5 \mathrm{~g}$ \\
Distilled water & $1 \mathrm{~L}$ \\
AAC Developer & \\
Solution A & \\
Ascorbic acid & $18 \mathrm{~g}$ \\
Distilled water & $0.25 \mathrm{~L}$ \\
Solution B & \\
Sodium carbonate (anhydrous) & $60 \mathrm{~g}$ \\
Distilled water & $0.75 \mathrm{~L}$ \\
Bleach Formula ${ }^{b}$ & \\
Solution A & \\
Ammonium dichromate & $20 \mathrm{~g}$ \\
Sulfuric acid & $14 \mathrm{~mL}$ \\
Distilled water & $1 \mathrm{~L}$ \\
Solution B & \\
Potassium bromide & $92 \mathrm{~g}$ \\
Distilled water & $1 \mathrm{~L}$ \\
\hline
\end{tabular}

${ }^{a}$ Just before use, mix A with B.

${ }^{b}$ Just before use, mix 1 part A with 10 parts distilled water; then add 30 parts $B$.

10. Dry in dissecator for $24 \mathrm{~h}$ at $20^{\circ} \mathrm{C}$ and $\mathrm{RH}<20 \%$.

This processing is a modification of the processing presented in Ref. 11: hypersensitizing the plates before exposure, increasing the time for washing the plates after development and bleaching (to be sure to eliminate residual chemicals in the layer), increasing the temperature of the bleach bath from $50{ }^{\circ} \mathrm{C}$ to $70{ }^{\circ} \mathrm{C}$, and drying the processed plates in a dessicator at low $\mathrm{RH}$ for $24 \mathrm{~h}$. instead of using a vacuum chamber. Increasing the temperature of the bleach bath is beneficial to softening the gelatin, which as we explained above is quite hard in the case of BB-640 plates. The developer used in these experiments was Kodak D-19, a nontanning developer, although it is important to note that oxidation products of this developer produce an important tanning action. ${ }^{7}$ The developing time was $5 \mathrm{~min}$.

The diffraction efficiency $\eta$ of the recorded holograms was calculated as the ratio of the diffracted beam intensity to the incident collimated probe-beam intensity of the $\mathrm{He}-\mathrm{Ne}$ laser, taking into account Fresnel losses and absorption due to the glass substrate. The efficiency of zero-order or transmission $\tau$ was similarly calculated as the ratio of the directly transmitted beam intensity to the incident power, corrected by taking into account losses due to reflection at different interfaces and absorption of the glass substrate. Figure 3 shows the variation of diffraction efficiency and minimum transmission for reconstruction under Bragg condition and for BB-640 and Agfa 8E75 HD plates. Both BB-640 and Agfa 8E75 HD plates were hypersensitized and processed fol-
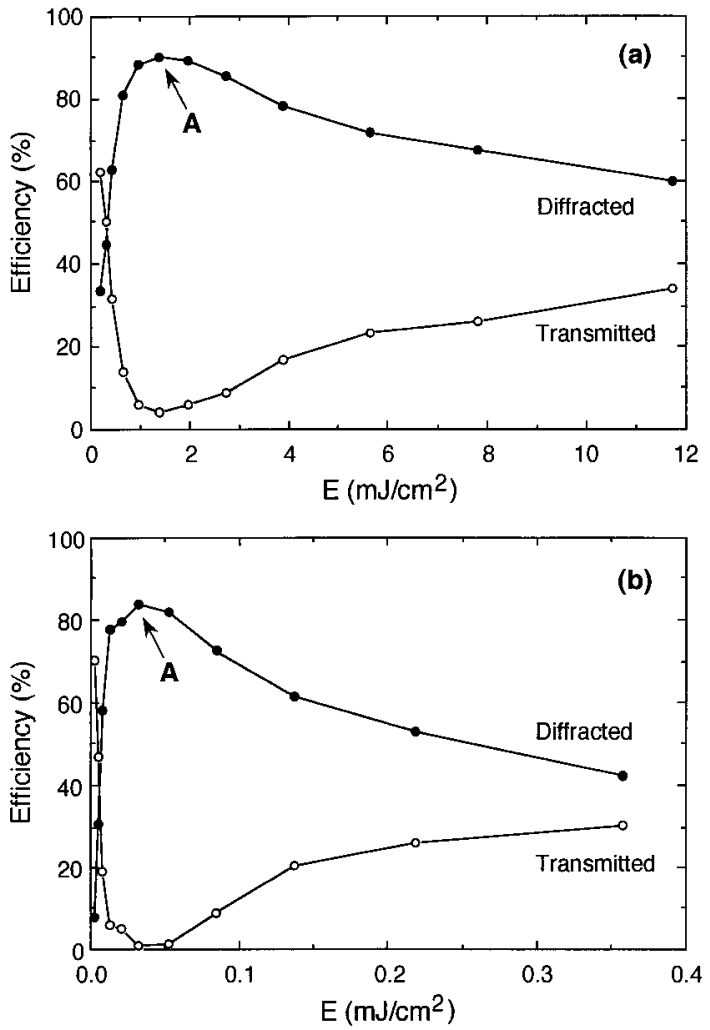

Fig. 3. Measured diffracted and transmitted beam intensities versus exposure under Bragg condition and for hypersensitized (a) BB-640 and (b) Agfa 8E75 HD plates developed with D-19.

lowing processes given above. As can be seen, BB640 shows better results with a diffraction efficiency of $90.3 \%$ (79\% if we do not consider Fresnel losses and the absorption of the glass plate) and a zero-order efficiency of $4.3 \%$. This implies that, of the light incident on the emulsion, only $5.4 \%$ remains unaccounted for, lost through scatter and absorption. However, for Agfa 8E75 HD plates processed under the same conditions, diffraction efficiency is $84 \%$, transmission is $0.7 \%$, and light unaccounted for is $15.3 \%$, almost three times more than for BB-640 plates. From the experimental data it can be seen that the highest diffraction efficiencies are obtained with hypersensitized BB-640 plates.

\section{Comparison of Theory with Experiment}

The efficiency of the diffracted and the transmitted beams, $\eta$ and $\tau$, respectively, together with the absorption of the material $\alpha d$ (where $\alpha$ is the absorption coefficient and $d$ is the thickness of the photographic emulsion after processing) are three parameters that may be used to characterize holographic transmission gratings recorded in silver halide sensitized gelatin of BB-640 and Agfa 8E75 HD emulsions. By means of Kogelnik's coupled-wave theory, ${ }^{27}$ it is possible to obtain an analytical expression for the diffraction efficiency of volume holograms.

The equation for diffraction efficiency $\eta$, given by 
Kogelnik's theory for phase transmission holographic gratings, is ${ }^{27}$ :

$$
\eta=\exp \left(-\alpha d / \cos \theta^{\prime}\right) \frac{\sin ^{2}\left(v^{2}+\xi^{2}\right)^{1 / 2}}{1+\left(\xi^{2} / v^{2}\right)},
$$

where for gratings with unslanted fringes $v$ and $\xi$ are expressed as follows:

$$
\begin{aligned}
& \nu=\frac{\pi \Delta n d}{\lambda \cos \theta^{\prime}}, \\
& \xi=\frac{\pi d}{\Lambda \cos \theta^{\prime}}\left(\left|\sin \theta^{\prime}\right|-\frac{\lambda}{2 n \Lambda}\right) .
\end{aligned}
$$

In Eqs. (7)-(9) $\alpha$ takes into account the absorption (and also the scatter; we have no means of differentiating between the two at this point), $d$ is the thickness, $n$ is the mean refractive index (we took the value of 1.54 for gelatin ${ }^{23}$ ), $\Delta n$ is the index modulation, $\Lambda$ is the grating period, $\lambda$ is the wavelength of reconstruction in air, and $\theta^{\prime}$ is the angle of reconstruction in the medium, which is related to the angle of reconstruction in air $\theta$, by Snell's law. For unslanted gratings the grating period $\Lambda$ is given by the equation

$$
\Lambda=\left(\lambda / 2 \sin \theta_{0}\right),
$$

where $2 \theta_{0}$ is the angle between the two beams in air. For gratings analyzed in this paper, $\theta_{0}=22.5^{\circ}$ and $\Lambda$ $=0.827 \mu \mathrm{m}$. As Kogelnik's theory is limited to the description of two diffraction orders, then

$$
\eta+\tau=\exp \left(-\alpha d / \cos \theta^{\prime}\right)
$$

and $\tau$ can be obtained as follows:

$$
\tau=\exp \left(-\alpha d / \cos \theta^{\prime}\right)-\eta,
$$

where $\tau$ is the transmission of the hologram. With Eq. (7) the value of $\tau$ may be calculated by means of the following equation:

$$
\tau=\exp \left(-\alpha d / \cos \theta^{\prime}\right)\left[1-\frac{\sin ^{2}\left(v^{2}+\xi^{2}\right)^{1 / 2}}{1+\left(\xi^{2} / \nu^{2}\right)}\right] .
$$

We tested the holograms in Fig. 3 (marked A in this figure) that exhibited high peak diffraction efficiency more extensively by rotating them, and the variation of transmission with the angle of incidence $\theta$ (in air) is shown in Fig. 4 for both BB-640 and Agfa 8E75 HD plates. The values of transmission $\tau$ were corrected to take into account Fresnel's reflections and the absorption of the glass substrate.

Before we evaluate the results obtained with Kogelnik's theory, some interesting conclusions may be drawn from an initial analysis of the experimental data shown in Fig. 4. According to Eqs. (9) and (13), the width of the transmittance curves is determined by the thickness $d$; thus the thicker the emulsion, the narrower the curves. Therefore it can be concluded from Fig. 4 that the emulsion of BB-640 plates is thicker than that of Agfa 8E75 HD plates. Also, the
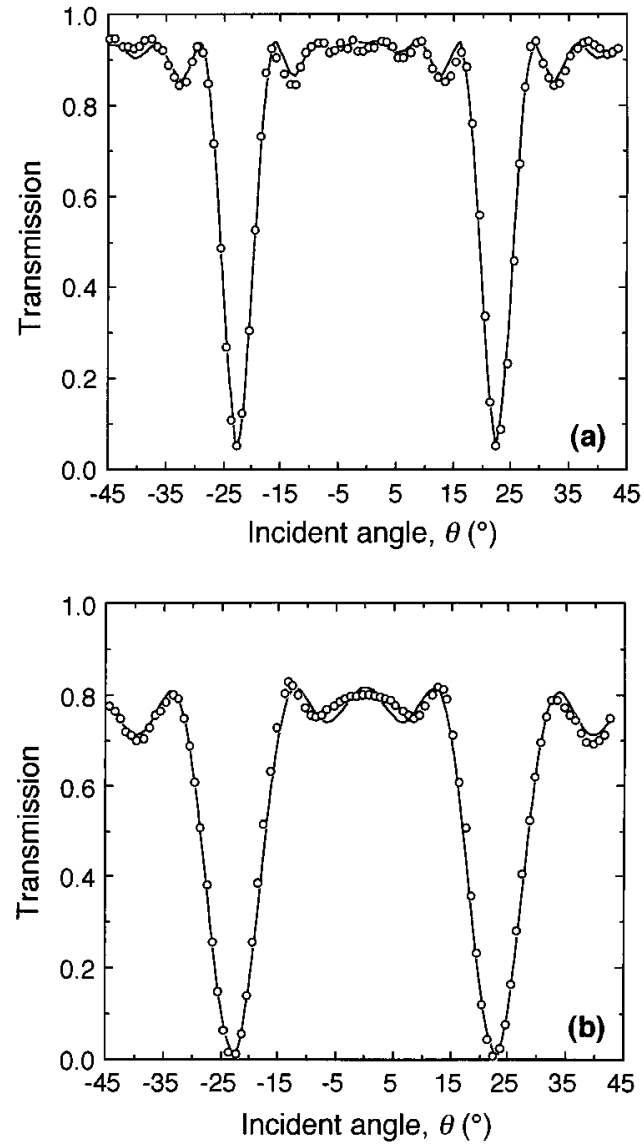

Fig. 4. Experimental measurements and theoretical fit (solid curve) of transmission as a function of the reconstruction angle in air for pretreated plates developed with D-19: (a) BB-640 emulsion, (b) Agfa 8E75 HD emulsion.

greater the absorption of the emulsion, the greater the value of the factor $\exp \left(-\alpha d / \cos \theta^{\prime}\right)$, and the maximum transmission will be lower outside Bragg condition. It is obvious from Fig. 4 that Agfa 8E75 HD plates have a higher absorption than BB-640 plates.

The theoretical curve of Eq. (13) was then fitted with the experimental data. Model parameters were altered to improve the agreement between theory and experiment. Modifying the values of $\Delta n$ (which largely determines the depth of the Bragg trough at Bragg angle), $d$ (which determines the width of the Bragg peaks and troughs), and $\alpha d$ (which takes into account the value of maximum transmission), the theoretical fit (solid curves in Fig. 4) was obtained with the values of the parameters shown in Table 3. The parameters obtained with this theoretical fit are in agreement with previous conclusions regarding thickness and absorption of the emulsions, with BB-640 plates showing less absorption and larger thickness.

\section{Influence of the Developer}

Developing is one of the most important steps in the processing of photographic emulsions. During developing, the latent image recorded when the plate is exposed is converted into an image formed of silver 
Table 3. Parameters of Holographic Gratings Recorded in BB-640 and Agfa 8E75 HD Emulsions ${ }^{a}$

\begin{tabular}{lcc}
\hline Parameter & BB-640 & Agfa 8E74 HD \\
\hline$d(\mu \mathrm{m})$ & 10.7 & 6.0 \\
$\alpha d$ & 0.062 & 0.200 \\
$\alpha\left(\mu \mathrm{m}^{-1}\right)$ & $5.8 \times 10^{-3}$ & $33.3 \times 10^{-3}$ \\
$\Delta n$ & 0.024 & 0.048 \\
\hline
\end{tabular}

${ }^{a}$ With D-19 developer for exposure giving maximum diffraction efficiency.

grains. There are many different developers used in holography, and each one has a different effect on the processing. In this paper we study the effect of two of these developers, D-19 and AAC. D-19 is a highcontrast, low fog developer that in general produces clear transmission holograms with most of existing photographic materials. It is more energetic than AAC, although it creates oxidation products that give rise to a lower resolution, and when the spatial frequency increases, the diffraction efficiency decreases considerably. The developing agent of AAC developer is ascorbic acid, which forms practically colorless oxidation products. It also has the advantage of not being toxic and moreover produces high contrast and a low fog level. ${ }^{28}$

Figure 5 shows the $D-\log E$ curves for D-19 and AAC developers with BB-640 plates hypersensitized according to the hypersensitization process given above and for an intensity of $500 \mu \mathrm{W} \mathrm{cm}{ }^{-2}$. Processing of absorption holograms with AAC developer was identical to that of holograms developed with D-19, except that the developing time was reduced to $4 \mathrm{~min}$. It can be seen that the slope of the linear zone of the $D-\log E$ curves is steeper for D-19 developer than for AAC. This allows us to state, in accordance with the explanation above, that D-19 developer gives a higher contrast. D-19 developer has a higher sensitivity than AAC, and the values of $S$ for hypersensitized BB-640 plates are $84 \mu \mathrm{J} \mathrm{cm}{ }^{-2}$ and $94 \mu \mathrm{J} \mathrm{cm} \mathrm{cm}^{-2}$ for D-19 and AAC developers, respectively. Also, a slightly higher maximum density is achieved with D-19 developer than with AAC developer.

Next, transmission holographic gratings of a spa-

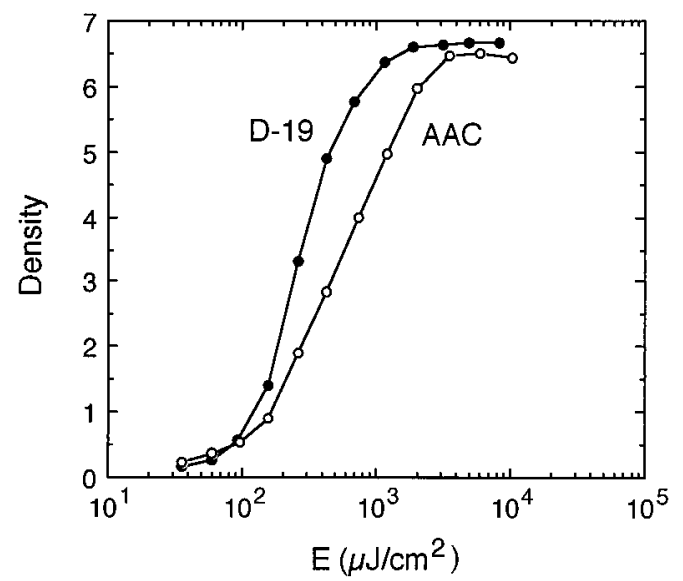

Fig. 5. $D-\log E$ curves for pretreated BB-640 emulsion and for D-19 and AAC developers.

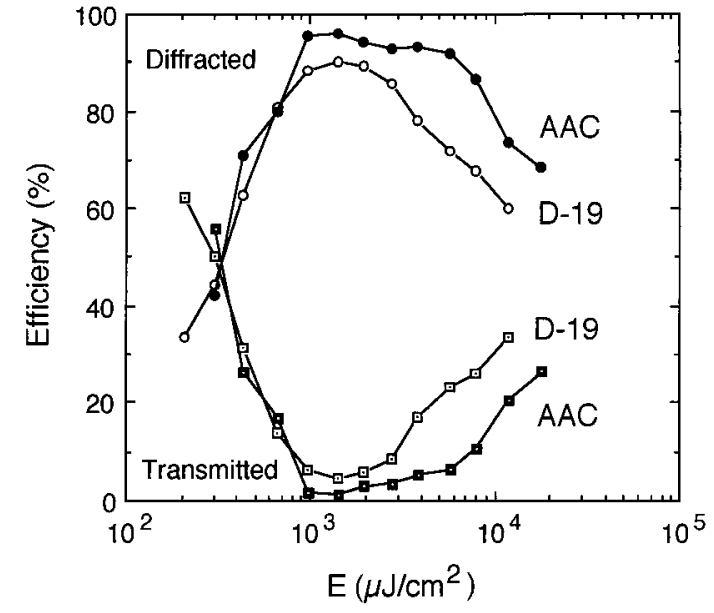

Fig. 6. Measured diffracted and transmitted beam efficiencies versus exposure for pretreated BB-640 plates and for two developers: D-19 and AAC. Reconstruction was performed at Bragg angle.

tial frequency of 1200 lines $\mathrm{mm}^{-1}$ were recorded and processed following the enumerated processing schedule given above, with hypersensitized BB-640 plates but with AAC developer instead of D-19 at $20^{\circ} \mathrm{C}$ and with a developing time of $4 \mathrm{~min}$. Figure 6 shows the variation of peak diffraction efficiency and minimum transmission with exposure for D-19 and AAC developers. As can be seen, AAC developer shows better results with a diffraction efficiency of 96.2\% (84.2\% if we do not consider Fresnel losses and the absorption of the glass backing) and a zero-order efficiency of $1.0 \%$, and then, of the light incident on the hologram, only $2.8 \%$ remains unaccounted for, lost through scatter and absorption. The exposure at which maximum efficiency is obtained is $1.4 \mathrm{~mJ}$ $\mathrm{cm}^{-2}$, approximately 350 times less than the exposure necessary for obtaining maximum diffraction efficiency in red-sensitive dichromated gelatin. ${ }^{29}$ For D-19 developer we obtained a diffraction efficiency of $90.3 \%$ and a transmission of $5.4 \%$, and light unaccounted for was $4.3 \%$. Moreover, the range of exposures at which high efficiencies are obtained is greater for AAC than for D-19, since diffraction efficiencies greater than $90 \%$ are obtained for exposures

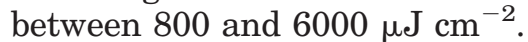

The percentage of absorption and scattering was calculated from diffraction efficiency $\eta$ and transmission $\tau$ with $100-\eta-\tau$, both measured at Bragg angle. Figure 7(a) shows the results for absorption and scatter losses for hypersensitized plates developed with D-19 and AAC developers. Figure 7(b) shows the results for scattered light intensity (in arbitrary units) measured with the setup shown in Fig. 8. As can be seen in Figure 7(b), the scattered light intensity is almost the same for D-19 and AAC developers, the only difference being that the graph corresponding to AAC is displaced with respect to that of D-19 along the exposure axis. This shift occurs because the energetic sensitivity of D-19 is greater than that of AAC. As can be seen in Fig. 7(a), the results obtained for absorption and scatter 

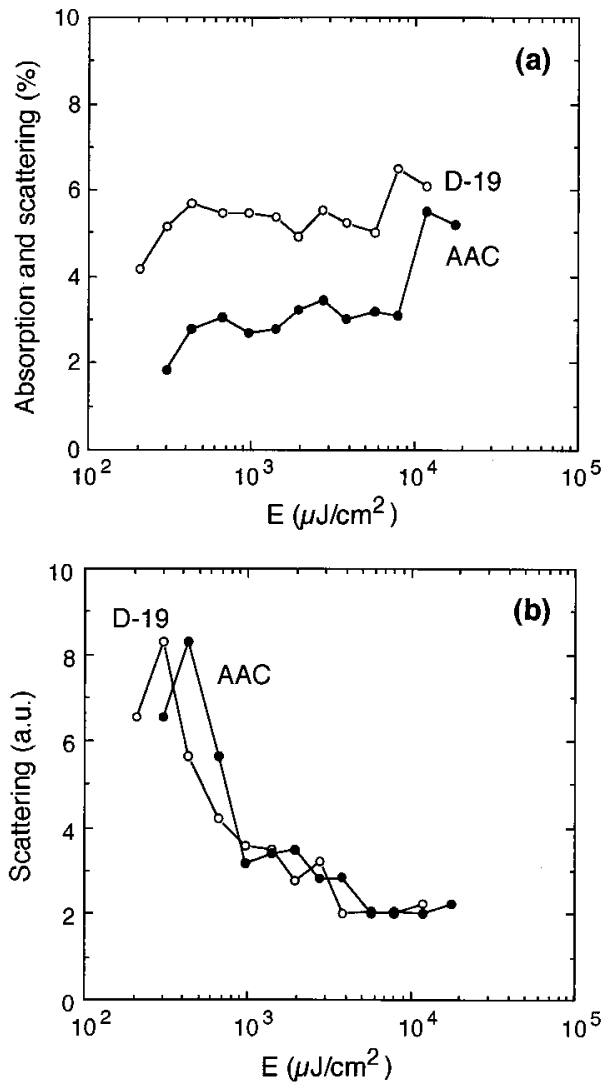

Fig. 7. Variation of (a) the absorption and scattering and (b) the scattered light intensity with exposure for SHSG transmission gratings recorded with pretreated BB-640 plates and for two developers: D-19 and AAC.

losses are similar, with regard to the shape of the graph and the dependence on exposure, for both developers. However, the results corresponding to AAC are lower than those for D-19, with values between $1.8 \%$ and $5.5 \%$ for AAC and between $4.2 \%$ and $6.5 \%$ for D-19. Since in Fig. 7(b) the scattering is similar for both developers, Fig. 7(a) indicates that the absorption is greater for holograms developed with D-19, since the oxidation products of this devel-

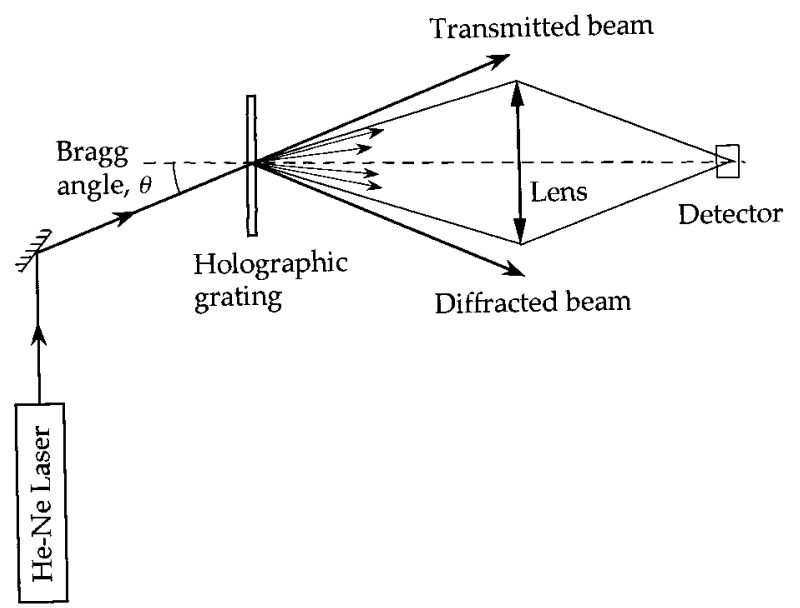

Fig. 8. Experimental setup for the measurement of scattered radiation.
Table 4. Characteristics of Holographic Gratings Recorded in BB-640 Photographic Emulsion ${ }^{a}$

\begin{tabular}{lcc}
\hline \multicolumn{1}{c}{ Parameter } & D-19 & AAC \\
\hline Diffracted beam $(\%)$ & $90.3 \pm 0.8$ & $96.2 \pm 0.8$ \\
Transmitted beam $(\%)$ & $4.3 \pm 0.04$ & $1.0 \pm 0.02$ \\
Absorption and scattering $(\%)$ & $5.4 \pm 0.8$ & $2.8 \pm 0.8$ \\
Scattering & 3.5 & 3.4 \\
\hline
\end{tabular}

${ }^{a}$ With D-19 and AAC developers for exposure giving maximum diffraction efficiency.

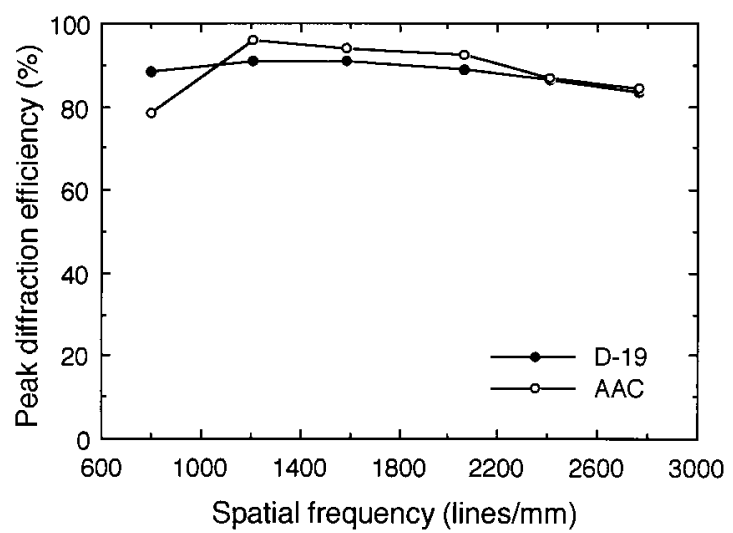

Fig. 9. Spatial-frequency response for hypersensitized BB-640 emulsion and for AAC and D-19 developers.

oper give rise to a greater absorption, owing to a small degree of coloring of the plate. Table 4 shows the comparative results for these two developers, corresponding in each case to the value of exposure that gives rise to maximum diffraction efficiency.

The diffraction efficiency as a function of the spatial-frequency curves is shown in Fig. 9 for both D-19 and AAC. The losses caused by Fresnel's reflections and the absorption due to the glass backing were accounted for. The spatial-frequency response is almost flat within the region of interest for D-19. However, for low spatial frequencies the results obtained with AAC developer are inferior to those obtained with D-19, whereas for other spatial frequencies the results obtained with both developers are similar. For D-19 the diffraction efficiency is greater than $83 \%$ (after allowing for reflections) for spatial frequencies between 800 and 2800 lines $\mathrm{mm}^{-1}$ (interbeam angles between $29.4^{\circ}$ and $122.2^{\circ}$ ). The spatial-frequency response for SHSG holograms recorded in BB-640 plates is better than for Agfa 8E75 HD plates, since with the latter plates the diffraction efficiency falls rapidly when the spatial frequency is increased. ${ }^{30}$ This rapid decrease in the diffraction efficiency observed with Agfa plates occurs because the grains are larger ${ }^{31}$ than those of BB-640 plates, as we indicated in Subsection 2.B.

\section{Conclusions}

We have described the processing techniques for SHSG in BB-640 emulsion, taking into account the different types of gelatin and the smaller grain size with respect to Agfa 8E75 HD plates, and we have 
analyzed the holographic characteristics, such as energetic and spectral sensitivities, diffraction efficiency, minimum transmission, absorption and scatter losses, scattered light intensity, and spatialfrequency response. The enumerated hypersensitization process gives our optimized processing, with which we have achieved a maximum diffraction efficiency of $96.2 \%$ (after allowing for reflections) and absorption and scatter losses of $2.8 \%$. These results confirm the application of BB-640 emulsion for recording high-quality transmission holograms in SHSG. Coupled-wave theory has given good agreement with experimental results, and information about the thickness, the absorption coefficient, and the modulation of the refractive index has been obtained. The high diffraction efficiency and low absorption and scatter obtained certainly opens new opportunities with regard to results obtained in experiments with SHSG holograms over the past few years. We believe the results presented in this paper to be some of the best reported for this type of processing technique at present, and they confirm the future application of SHSG derived from BB-640 plates for recording high-quality phase transmission holograms. The results also show that BB-640 plates are a potential replacement for Agfa 8E75 HD emulsion at a crucial moment when, since Agfa has ceased its production of holographic material, it is necessary to find alternative silver halide materials. Finally, the information presented in this paper with regard to the performance of BB-640 plates might be useful to researchers working in the areas of display holograms, holographic optical elements, and holographic data storage.

This study was supported by the Comisión Interministerial de Ciencia y Tecnología of Spain under project MAT97-0705-C02-02.

\section{References}

1. H. I. Bjelkhagen, Silver Halide Recording Materials for Holography and Their Processing (Springer-Verlag, Berlin, 1993), pp. 252-262.

2. P. Hariharan, Optical Holography (Cambridge University, London, 1996), pp. 111-112.

3. K. S. Pennington and J. S. Harper, "Techniques for producing low-noise, improved efficiency holograms," Appl. Opt. 9, 16431650 (1970).

4. W. R. Graver, J. W. Gladden, and J. W. Eastes, "Phase holograms formed by silver halide (sensitized) gelatin processing," Appl. Opt. 19, 1529-1536 (1980).

5. B. J. Chang and K. Winick, "Silver-halide gelatin holograms," in Recent Advances in Holography, T. C. Lee and P. N. Tamura, eds., Proc. Soc. Photo-Opt. Instrum. Eng. 215, 172-177 (1980).

6. R. A. Ferrante, "Silver halide gelatin spatial frequency response,” Appl. Opt. 23, 4180-4181 (1984).

7. P. Hariharan, "Silver halide sensitized gelatin holograms: mechanism of hologram formation," Appl. Opt. 25, 2040-2042 (1986).

8. D. K. Angell, "Improved diffraction efficiency of silver halide (sensitized) gelatin," Appl. Opt. 26, 4692-4702 (1987).

9. V. Weiss and E. Millul, "Bleached silver halide holographic recording materials," in Holography Techniques and Applica- tions, W. P. Jueptner, ed., Proc. Soc. Photo-Opt. Instrum. Eng. 1026, 55-61 (1988).

10. V. Weiss and A. A. Friesem, "Storage mechanism of volume phase holograms recorded in silver halide emulsions," J. Opt. Soc. Am. A 11, 2004-2010 (1994).

11. A. Fimia, A. Beléndez, and I. Pascual, "Silver halide (sensitized) gelatin in Agfa-Gevaert plates: the optimized procedure," J. Mod. Opt. 38, 2043-2045 (1991).

12. E. S. Simova and M. Kavehrad, "Silver-halide sensitized gelatin derived from Agfa-Gevaert holographic plates," Appl. Opt. 33, 1875-1879 (1994).

13. N. J. Phillips, R. D. Rallison, C. A. Barnett, S. R. Schicker, and Z. A. Colemann, "Dichromated gelatin: some heretical comments," in Practical Holography VII: Imaging and Materials, S. A. Benton, ed., Proc. Soc. Photo-Opt. Instrum. Eng. 1914, 101-114 (1993).

14. I. Pascual, A. Beléndez, and A. Fimia, "Reflection holographic optical elements in silver halide sensitized gelatin," Opt. Appl. 21, 239-244 (1991).

15. A. Fimia, I. Pascual, and A. Beléndez, "Silver halide sensitized gelatin as holographic recording material," Opt. Laser Technol. 27, 285-292 (1995).

16. E. S. Simova and M. Kavehrad, "Holographic $4 \times 4$ starcoupler in silver-halide sensitized gelatin," Opt. Eng. 32, 22332239 (1993).

17. N. J. Phillips, "Agfa-Gevaert departure: problem, or a new dawn for holography?" Holography Newsletter 8(1), 7 (1997).

18. R. Birenheide, "The BB series silver halide holographic recording material," Holography Newsletter 8(1), 12 (1997).

19. R. Birenheide, "BB emulsion series: current standings and future developments," in Sixth International Symposium on Display Holography, T. H. Jeong, ed., Proc. Soc. Photo-Opt. Instrum. Eng. 3358, 28-30 (1997).

20. N. J. Phillips, H. Heyworth, and T. Hare, "On Lippmann's photography," J. Photogr. Sci. 32, 158-169 (1984).

21. H. I. Bjelkhagen, Silver Halide Recording Materials for Holography and Their Processing (Springer-Verlag, Berlin, 1993), p. 107.

22. A. Kozma, "Effects of film-grain noise in holography," J. Opt. Soc. Am. 58, 436-438 (1968).

23. H. I. Bjelkhagen, Silver Halide Recording Materials for Holography and Their Processing (Springer-Verlag, Berlin, 1993), pp. 82-83.

24. R. K. Kostuk and J. W. Goodman, "Refractive index modulation mechanism in bleached silver halide holograms," Appl. Opt. 30, 369-371 (1991).

25. A. Beléndez, C. Neipp, M. Flores, and I. Pascual, "Highefficiency silver-halide sensitized gelatin holograms with low absorption and scatter," J. Mod. Opt. 45, 1985-1992 (1998).

26. H. I. Bjelkhagen, Silver Halide Recording Materials for Holography and Their Processing (Springer-Verlag, Berlin, 1993), pp. 18-19.

27. H. Kogelnik, "Coupled wave theory for thick hologram gratings,” Bell Syst. Tech. J. 48, 2909-2947 (1969).

28. J. Crespo, A. Fimia, and J. A. Quintana, "Fixation-free methods in bleached reflection holography," Appl. Opt. 25, 16421645 (1986).

29. R. Changkakoti and S. V. Pappu, "Towards optimum diffraction efficiency for methylene blue sensitized dichromated gelatin holograms," Opt. Laser Technol. 21, 259-263 (1989).

30. A. Fimia, I. Pascual, and A. Beléndez, "Optimized spatial frequency response in silver halide sensitized gelatin,” Appl. Opt. 31, 4625-4627 (1992).

31. L. Joly and R. Vanhorebeek, "Development effects in whitelight reflection holography," Photogr. Sci. Eng. 24, 108-113 (1980). 